\title{
Efeitos do Glyphosate e Sulfometuron-Methyl no Crescimento E Na Qualidade Techológica da CaNa-de-Açúcar ${ }^{1}$
}

\author{
Effect of Glyphosate and Sulfometuron-Methyl on the Growth and Technological Quality of \\ Sugarcane
}

MESCHEDE, D.K. ${ }^{2}$, VELINI, E.D. ${ }^{3}$ e CARBONARI, C.A. ${ }^{4}$

\begin{abstract}
RESUMO - Os herbicidas, mesmo quando usados em doses reduzidas ou utilizados como maturadores, podem alterar a morfofisiologia da planta, o que pode levar a modificações qualitativas e quantitativas na produção. O presente estudo objetivou avaliar a eficiência agronômica e os efeitos, durante o crescimento da cana-soca, da aplicação de glyphosate e sulfometuron-methyl em baixas doses. O delineamento experimental utilizado foi o de blocos casualizados, com quatro repetições. Os tratamentos foram constituídos pelos herbicidas sulfometuron-methyl e glyphosate em diferentes doses e misturas e por uma testemunha (sem aplicação dos produtos). Uma linha de plantas de cana-de-açúcar foi destinada à aferição da qualidade tecnológica, sendo estabelecido $1 \mathrm{~m}$ aleatório a cada época de amostragem. Os colmos coletados foram submetidos ao desponte na altura da gema apical e à desfolha; em seguida, foram encaminhados para processamento segundo a metodologia do Sistema de Pagamento de Cana pelo Teor de Sacarose (SPCTS), sendo considerados os parâmetros tecnológicos: pol cana (PCC), pureza do caldo (PUI), açúcar total recuperável (ATR) e Brix. Nas soqueiras de cana-de-açúcar, realizaram-se análises de crescimento (altura e perfilhos). As avaliações foram realizadas na pré-colheita (30 dias após aplicação dos maturadores) e 30, 60, 90, 120, 150 e 180 dias após a colheita. Os herbicidas glyphosate e sulfometuronmethyl propiciaram melhoria da qualidade tecnológica da matéria-prima, com incrementos significativos na pureza do caldo e no Brix. A aplicação dos produtos não interferiu na produtividade e no teor de açúcar. Houve efeito estimulante no perfilhamento quando se usou glyphosate na dose de $400 \mathrm{~mL} \mathrm{ha}^{-1}$ e redução em crescimento (altura) no início do desenvolvimento da cana, porém, com o tempo, o efeito não se manteve.
\end{abstract}

Palavras-chave: Sacharum spp., fisiologia da cana-de-açúcar, maturadores de crescimento.

\begin{abstract}
Even when used at low doses, or used as growth regulators, herbicides can change the morphology and physiology of the plant, leading to qualitative and quantitative changes in production. This study aimed to evaluate the agronomic efficiency and the effects during sugarcane ratoon growth after applying low doses of glyphosate and sulfometuron-methyl. The experiment was arranged in a randomized block design with four replications. The treatments consisted of application of two herbicides (glyphosate and sulfometuron-methyl at various doses and mixtures) and a control (without herbicide application). One row of plants was used to measure technological quality, with a random $1 \mathrm{~m}$ being established for each sampling time. The stems collected were submitted to apical bud emergence and defoliation and were sent to be processed according to the methodology of the Cane Payment System based on Sucrose Content (CPSSC), considering the following technological parameters: pol cane $(P C C)$, broth purity (BP), total recoverable sugar (TRS), and Brix. The stumps were analyzed for sugarcane growth (height and tillers). Evaluations were performed during pre-harvest (30 DAA), 30, 60, 90, 120, 150 and 180 days after harvest. The treatments with glyphosate and sulfometuronmethyl provided improved technological quality of the raw material, with significant increases in broth purity and Brix. Herbicide application did not affect productivity and sugar content. There was a stimulating effect on tillering with glyphosate at a dose of $400 \mathrm{~mL}$, and growth reduction (height) at the start of cane development, but such effect was not maintained along time.
\end{abstract}

Keywords: Saccharum spp., sugarcane, growth regulators.

1 Recebido para publicação em 21.11.2009 e na forma revisada em 17.12.2010.

2 Pesquisadora, Dra., Dep. de Produção Vegetal, Faculdade de Ciências Agronômicas - FCA/UNESP, Caixa Postal 237, 18603-970, Botucatu-SP, <meschede@fca.unesp.br>; ${ }^{3}$ Professor, Dr., Dep. de Produção Vegetal, FCA/UNESP, <velini@fca.unesp.br>; ${ }^{4}$ Engo-Agrōo., Dr., Dep. de Produção Vegetal, FCA/UNESP, <carbonari@fca.unesp.br>. 


\section{INTRODUÇÃO}

O rendimento econômico da cana-deaçúcar é medido pela produção de sacarose (componente mais valioso), além de açúcares não redutores, utilizados para formar o melaço e também a fibra, que pode ser usada como fonte de energia para a própria usina.

A produtividade média de cana-de-açúcar tem oscilado entre 90 e 120 toneladas de matéria vegetal por hectare, porém, adotandose manejo adequado de variedades, de calagem e de adubação, podem-se alcançar produtividades superiores a 150 toneladas por hectare (Oliveira et al., 2002; Cosecana, 2003).

O processo de maturação da cana-de-açúcar na região Sudeste do Brasil ocorre naturalmente a partir dos meses de abril a maio. Nesse período, as condições climáticas caracterizam-se pela gradativa queda da temperatura e diminuição das precipitações. Essas condições podem estimular o crescimento vegetativo da planta em detrimento do acúmulo de sacarose, implicando a geração de matéria-prima de qualidade inferior ou mesmo sua escassez para o funcionamento da indústria sucroalcooleira (Viana, 2008).

A disponibilidade de cultivares produtivos, com maturação precoce, pode ser viabilizada com a utilização de maturadores para o suprimento do complexo industrial (Caputo et al., 2008); mesmo sob condições climáticas que favoreçam a vegetação da cana-de-açúcar, também se pode induzir a maturação através da aplicação de produtos químicos (Castro et al., 2002).

Os produtos disponiveis no mercado apresentam ações distintas: alguns retardam e outros inibem o crescimento da cana-deaçúcar. Em ambos os casos, atuam sobre o balanço de fotoassimilados produzidos, favorecendo o acúmulo destes nos tecidos de reserva dos colmos, proporcionando assim incremento no teor de sacarose. Entretanto, considerandose os efeitos sobre os resultados industriais, especialmente os que se referem aos processos fermentativos, muitas discussões têm surgido envolvendo o emprego de maturadores quimicos na cultura. Por exemplo, em estádios finais do ciclo há relatos da ocorrência de reflexos negativos sobre o desenvolvimento do processo e também da viabilidade dos microrganismos, além da dificuldade de manter a força do fermento no início da fase de multiplicação, quando se usam os maturados.

Há vários trabalhos que indicam o glyphosate como uma alternativa técnica e econômica que permite flexibilizar o manejo de corte e manejar o comportamento das variedades de cana-de-açúcar. Segundo resultados de pesquisas, observaram-se a melhoria na qualidade da matéria-prima para a indústria, a paralisação do florescimento, a otimização do potencial de maturação das variedades e a maximização da margem de contribuição agrícola e industrial quando se usou esse herbicida como maturador (Castro et al., 2002; Almeida et al., 2003).

O presente trabalho teve por objetivo avaliar o efeito da aplicação de herbicidas utilizados como maturadores de cana-de-açúcar e a influência na qualidade industrial da cultura em pré-colheita e na cana-soca.

\section{MATERIAL E MÉTODOS}

O experimento foi conduzido em cana-soca de segundo corte, variedade SP 803280, na fazenda Jurema, no município de Igaraçu do Tietê, Estado de São Paulo (latitude de 22 $38^{\circ}$ $45^{\prime}$ 'S, longitude de $48^{\circ} 36^{\prime}$ ' 29'W e altitude de $620 \mathrm{~m}$ ), pertencente ao Grupo COSAN Unidade Barra.

O delineamento experimental adotado foi de blocos ao acaso, com quatro repetições. Cada parcela foi constituída de $160 \mathrm{~m}^{2}$ (1,6 m entre plantas, em 10 linhas, e $10 \mathrm{~m}$ de comprimento), totalizando uma área de $960 \mathrm{~m}^{2}$. Para área útil foram consideradas as cinco linhas centrais. Os tratamentos constituíram-se da aplicação dos herbicidas sulfometuron-methyl e glyphosate, que são encontrados comercialmente como Curavial (750 $\left.\mathrm{g} \mathrm{kg}^{-1}\right)$ e Roundup original $\left(360 \mathrm{~g} \mathrm{~L}^{-1}\right)$. As doses utilizadas foram: glyphosate: 200 e $400 \mathrm{~mL}$ de p.c. ha ${ }^{-1}$; sulfometuron-methyl $20 \mathrm{~g} \mathrm{ha}^{-1}$ sem a adição de adjuvantes; as misturas de glyphosate + sulfometuron-methyl em $200+10,150+$ $12 \mathrm{~g} \mathrm{ha}^{-1}$ de produtos comerciais, respectivamente; e mais uma testemunha.

A aplicação dos produtos foi realizada em $11 / 10 / 2006$, utilizando equipamento costal 
pressurizado $\left(\mathrm{CO}_{2}\right)$ com barra de $6 \mathrm{~m}$ de comprimento, contendo seis pontas de pulverização AXI 11002, sendo a pressão de trabalho de 50 psi, o que proporcionou a vazão de $100 \mathrm{~L} \mathrm{ha}^{-1}$. A umidade relativa do ar no momento da aplicação era de $80 \%$, com velocidade do vento de $6 \mathrm{~m} \mathrm{~s}^{-1}$ e temperatura média de $25^{\circ} \mathrm{C}$.

As avaliações de pré-colheita foram realizadas aos 15 e 30 dias após aplicação dos maturadores. Foram avaliados parâmetros tecnológicos da cana-de-açúcar, compostos pelo TCH (tonelada de colmos por hectare), TPH (tonelada de Pol por hectare) POL.(\% de massa de sacarose aparente), Brix (\% de massa de sólidos solúveis contidos na solução de sacarose pura), PUC (pureza de caldo), ATR (açúcar total recuperável) e peso de bagaço úmido (PBU).

Uma linha de plantas foi destinada à aferição tecnológica, sendo estabelecido $1 \mathrm{~m}$ aleatório a cada época de amostragem. Os colmos coletados foram submetidos ao desponte na altura da gema apical e à desfolha e, em seguida, encaminhados para processamento segundo a metodologia do Sistema de Pagamento de Cana pelo Teor de Sacarose (SPCTS), descrita em Fernandes (2003).

A soqueira de cana-de-açúcar foi avaliada aos 30,60,90,120,150 e 180 dias após collheita, quando se coletou material para as análises de crescimento. Os parâmetros de crescimento foram: altura de plantas aferida da base do colmo, rente ao solo, até a folha +1 , ou seja, com a primeira lígula aberta; números de perfilhos, consistiu no levantamento do número de colmos por planta, de $1 \mathrm{em} 1 \mathrm{~m}$, perfazendo uma área de $10 \mathrm{~m}$ aleatório em cada parcela.

Os resultados foram submetidos à análise de variância pelo teste $F$; sendo significativos, aplicou-se o teste de $\mathrm{T}$ a $5 \%$ de probabilidade para comparação entre as médias. A comparação entre a testemunha e os tratamentos, por meio gráfico, foi realizada pelo cálculo de probabilidade, expresso pela fórmula: $(100 * 2 n-2 x)$, em que $x$ representa os valores que foram influenciados pelo tratamento, isto é, encontram-se acima da linha de tendência traçada pela equação, e y representa os valores abaixo da linha de tendência traçada pela equação, isto é, refletem os valores não influenciados pelos tratamentos.

\section{RESULTADOS E DISCUSSÃO}

Na Tabela 1, verificou-se que a produtividade tecnológica da cana de açúcar não foi afetada significativamente pela aplicação de glyphosate e sulfometuron-methyl, utilizados como maturadores. No entanto, a mistura de glyphosate $\left(200 \mathrm{~mL}+10 \mathrm{~g} \mathrm{ha}^{-1}\right)$ e de sulfometuron-methyl demonstrou ganho em produtividade de colmos de 9,3 $\mathrm{t} \mathrm{ha}^{-1} \mathrm{e}$ $14 \%$ a mais de teor de sacarose em relação à testemunha. O mesmo ocorreu para a TPH,

Tabela 1 - Produtividade de cana-de-açúcar medida por TCH (toneladas de colmos ha $\left.{ }^{-1}\right)$, TPH (toneladas de pol ha ${ }^{1}$ ) e pol (massa de sacarose aparente), avaliadas em pré-colheita. Igaraçu do Tiete-SP, 2006

\begin{tabular}{|c|c|c|c|}
\hline \multirow{2}{*}{ Tratamento } & \multicolumn{3}{|c|}{ Parâmetro de produtividade } \\
\hline & $\mathrm{TCH}$ & TPH & POL $(\%)$ \\
\hline 1. glyphosate ${ }^{1}$ a $200 \mathrm{~mL} \mathrm{ha}^{-1}$ & 72,8 & 11,3 & 16,2 \\
\hline 2. glyphosate ${ }^{1}$ a $400 \mathrm{~mL} \mathrm{ha}^{-1}$ & 73,3 & 11,6 & 15,8 \\
\hline 3. glyphosate ${ }^{1}$ a $200 \mathrm{~mL} \mathrm{ha}^{-1}+$ sulfometuron-methyl $^{2}$ a $10 \mathrm{~g} \mathrm{ha}^{-1}$ & 78,9 & 12,7 & 16,0 \\
\hline 4. glyphosate ${ }^{1}$ a $150 \mathrm{~mL} \mathrm{ha}^{-1}+$ sulfometuron-methyl $^{2}$ a $12 \mathrm{~g} \mathrm{ha}^{-1}$ & 75,8 & 12,5 & 16,5 \\
\hline 5. sulfometuron-methyl a $20 \mathrm{~g} \mathrm{ha}^{-1}$ & 74,6 & 12,1 & 16,2 \\
\hline 6. Testemunha & 69,6 & 10,5 & 14,3 \\
\hline F tratamento & $0,090^{\mathrm{ns}}$ & $0,077^{\mathrm{ns}}$ & $0,038^{\mathrm{ns}}$ \\
\hline F bloco & $0,0321^{\mathrm{ns}}$ & $0,15^{\mathrm{ns}}$ & $0,630^{\mathrm{ns}}$ \\
\hline CV $(\%)$ & 8,9 & 15,42 & 9,97 \\
\hline DMS & 11,01 & 3,05 & 8,27 \\
\hline
\end{tabular}

${ }^{\text {ns }}$ Médias não diferem estatisticamente pelo teste de $\mathrm{T}$ a $5 \%$ de probabilidade. 
tratando-se de um reflexo do TCH. Resultados semelhantes foram observados por Castro et al. (2002) e Viana (2008): a aplicação de maturadores não promoveu alterações significativas na produtividade de colmos em comparação com a testemunha. No entanto, Barbosa et al. (2007) e Leite et al. (2009) constataram incrementos significativos para produtividade de colmos.

Na Tabela 2 são apresentados os dados de Brix, ATR e PBU da cana-de-açúcar na pré-colheita. Não se verificaram diferenças significativas para valores de Brix e PBU da cana-de-açúcar com a aplicação dos produtos, quando comparados com os da testemunha. Os valores de ATR tiveram incrementos significativos em todos os tratamentos que receberam aplicação dos produtos, quando comparados com a testemunha. Esses resultados provavelmente se devem à maior influência que a sacarose apresenta sobre os açúcares totais. Corroborando os resultados obtidos, Romero et al. (2003) também verificaram que o glyphosate e sulfometuron-methyl induziram aumento de sacarose em todas as seções do colmo da cana-de-açúcar.

Segundo Casagrande (1991), o mecanismo de acúmulo de sacarose é o mesmo, tanto em tecidos imaturos como em adultos, ocorrendo: hidrólise da sacarose, como um pré-requisito e limitante da primeira etapa; formação e interconversão de hexosesfosfatos; formação de moléculas análogas à sacarose (talvez
sacarose-P); e acúmulo de parte da sacarose no vacúolo. Todavia, algumas diferenças entre os acúmulos nesses dois tecidos ocorrem, como a presença de reguladores vegetais e a ação das invertases.

No início do desenvolvimento da cana-deaçúcar, aos 30 DAC, observou-se paralisação do crescimento em altura na cana que recebeu aplicação do glyphosate, na dose de $400 \mathrm{~mL}$; no entanto, esse efeito não se manteve no decorrer das avaliações - aos 120 DAC, não se observou diferença entre os tratamentos (Figura 1). De acordo com Romero et al. (2000), doses elevadas de glyphosate, superiores a $0,40 \mathrm{~L} \mathrm{ha}^{-1}$, podem causar retardamento do processo de brotação e crescimento da cultura no ciclo seguinte, além de causar amarelecimento severo e dessecamento das plantas.

A inibição ou retardamento do crescimento da cana-de-açúcar, pela maior dose de glyphosate aplicada, pode ser devido à inibição da sintese do ácido indol acético (AIA). A inibição do alongamento do caule pode também estar relacionada á capacidade do AIA em promover síntese de etileno, pelo aumento da atividade 1-aminociclopropano-1-ácido carboxílico (ACC) (Liang et al., 1992). O aumento do etileno pode estimular o processo de senescência e a germinação das gemas laterais; ainda, o balanço hormonal entre o AIA e o etileno pode levar à inibição do alongamento do caule. Outra resposta pode ser a própria inibição da biossintese do AIA, pela rota

Tabela 2 - Parâmetros de produção e qualidade da cana-de-açúcar, medidas por meio do Brix (\% sólidos solúveis contidos na sacarose), ATR (açúcar total recuperável) e PBU (peso do bagaço úmido), avaliados na pré-colheita. Igaraçu do Tiete-SP, 2006

\begin{tabular}{|c|c|c|c|}
\hline \multirow{2}{*}{ Tratamento } & \multicolumn{3}{|c|}{ Parâmetro de produtividade } \\
\hline & Brix $(\%)$ & PBU & ATR \\
\hline 1. glyphosate ${ }^{1}$ a $200 \mathrm{mLl} \mathrm{ha}^{-1}$ & 18,0 & 158,5 & 152,0 \\
\hline 2. glyphosate ${ }^{1}$ a $400 \mathrm{~mL} \mathrm{ha}^{-1}$ & 17,5 & 154,8 & 153,0 \\
\hline 3. glyphosate ${ }^{1}$ a $200 \mathrm{~mL} \mathrm{ha}^{-1}+$ sulfometuron-methyl $^{2}$ a $10 \mathrm{~g} \mathrm{ha}^{-1}$ & 17,8 & 157,3 & 151,8 \\
\hline 4. glyphosate ${ }^{1}$ a $150 \mathrm{~mL} \mathrm{ha}^{-1}+$ sulfometuron-methyl ${ }^{2}$ a $12 \mathrm{~g} \mathrm{ha}^{-1}$ & 18,3 & 162,0 & 153,5 \\
\hline 5. sulfometuron-methyl ${ }^{2}$ a $20 \mathrm{~g} \mathrm{ha}^{-1}$ & 18,0 & 158,5 & 150,5 \\
\hline 6. Testemunha & 18,0 & 142,8 & 149,3 \\
\hline F tratamento & $2,40^{\text {ns }}$ & $1,08^{\text {ns }}$ & $1,39^{*}$ \\
\hline F bloco & $2,50^{\mathrm{ns}}$ & $0,53^{\mathrm{ns}}$ & $10,09^{*}$ \\
\hline $\mathrm{CV}(\%)$ & 1,86 & 8,30 & 1,76 \\
\hline DMS & 0,50 & 19,47 & 4,02 \\
\hline
\end{tabular}

${ }^{\text {ns }}$ Médias não diferem estatisticamente pelo teste de $\mathrm{T}$ a $5 \%$ de probabilidade. 

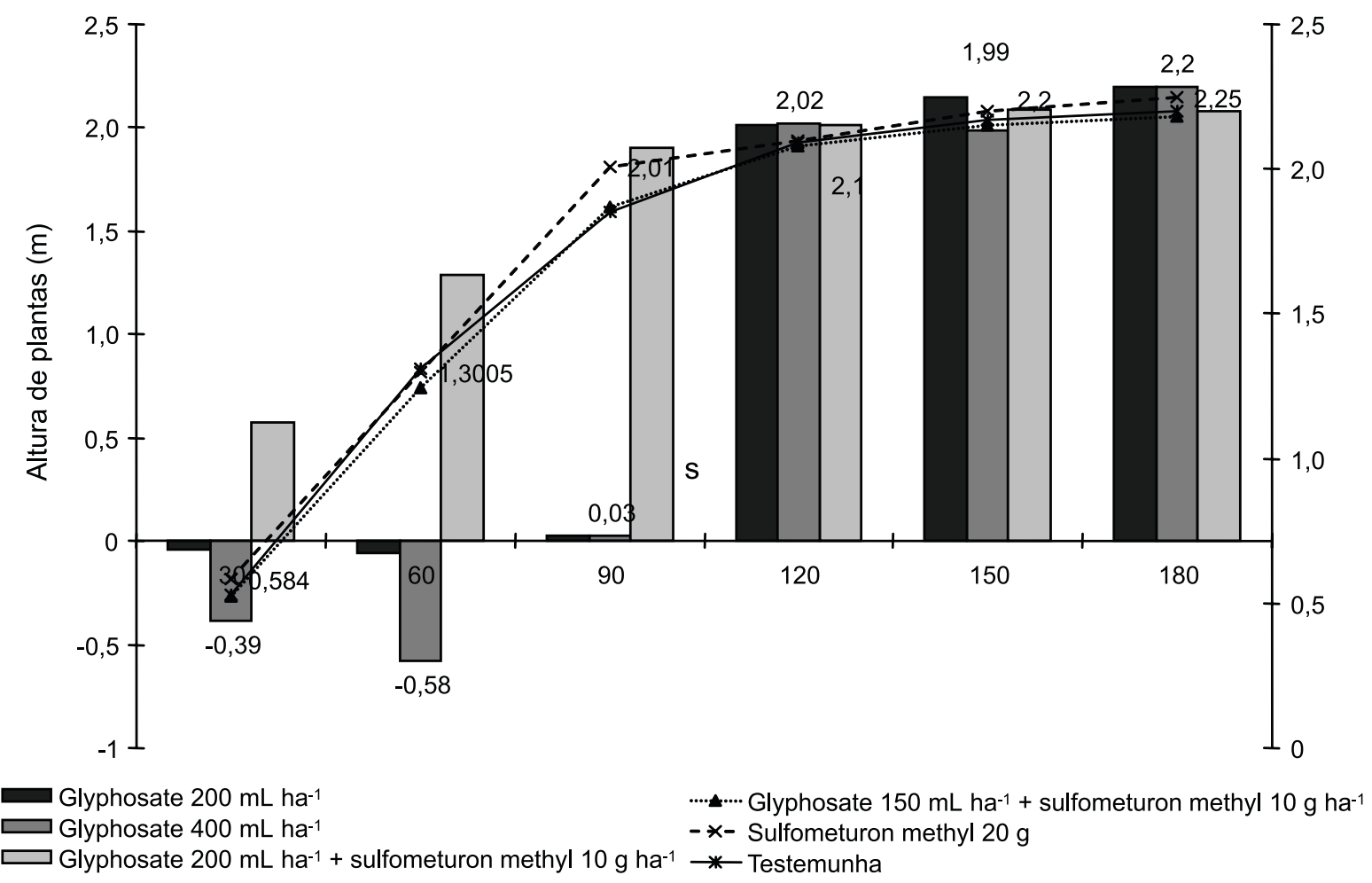

Figura 1 - Altura (metros) de plantas de cana-de-açúcar submetida à aplicação de diferentes tratamentos. Igaraçu do Tiete-SP, 2007.

independente do triptofano, e tem como precursor o indol 3-glicerol fosfato, que é dependente do corismato, o qual pode ser inibido pelo glyphosate na rota do ácido shiquímico.

Diversos trabalhos foram desenvolvidos para verificar o efeito de reguladores de crescimento, aplicados em pré (Wiedenfeld, 2003; Viana et al., 2008; Leite et al., 2009) ou pós-emergência (Shetiya \& Dendsay, 1991; Milhollon \& Legendre, 1995, na brotação e no desenvolvimento inicial da cana-de-açúcar, objetivando maior perfilhamento e produção de colmo final. Os resultados ainda são contraditórios. Conforme os dados apresentados na Tabela 3, os tratamentos com glyphosate promoveram incrementos significativos no número de perfilhos somente no início do crescimento da cana; depois disso, houve decréscimo, independentemente do tratamento, sem haver diferenças significativas. Resultado semelhante foi observado por Silva et al. (2007), que, ao trabalharem com genótipos de cana-de-açúcar, observaram a influência dos maturadores no perfilhamento da cana-soca no início do desenvolvimento.
Esse aumento no perfilhamento em até três meses de idade e posterior redução de cerca de $50 \%$, seguida de estabilização tanto em cana-planta quanto em cana-soca, a partir dos nove meses, é uma característica fisiológica da cana-de-açúcar já observada por vários autores (Barbieri, 2005; Castro \& Christoffoleti, 2005; Silva et al., 2007).

Como somente o glyphosate demonstrou efeito significativo sobre número de perfilhos (Figura 2) foram apresentados os efeitos da aplicação de glyphosate $400 \mathrm{~mL}$ e $200 \mathrm{~mL}$ em relação à testemunha. Na maior dose, houve estímulo no número de perfilhos, relação esta que não se manteve quando se reduziu a dose, ou quando se aplicou sulfometuron-methyl.

O glyphosate na maior dose, provavelmente, inibe a biossintese do AIA e, consequentemente, aumenta a sintese do etileno, promovendo estimulo na quebra das gemas dormentes, aumentando assim o número de perfilhos.

O glyphosate e sulfometuron-methyl, nas doses estudadas, melhoraram a qualidade 
Tabela 3 - Números de perfilhos por cana-de-açúcar submetidos a tratamentos com maturadores. Igaraçu do Tiete-SP, 2007

\begin{tabular}{|c|c|c|c|c|c|c|}
\hline \multirow{2}{*}{ Tratamento } & \multicolumn{6}{|c|}{ Perfilhos } \\
\hline & $30 \mathrm{DAC}$ & $60 \mathrm{DAC}$ & $90 \mathrm{DAC}$ & $120 \mathrm{DAC}$ & $150 \mathrm{DAC}$ & $180 \mathrm{DAC}$ \\
\hline 1. glyphosate ${ }^{1}$ a $200 \mathrm{~mL} \mathrm{ha}^{-1}$ & 36,5 & 39,75 & 36,8 & 22,5 & 20,5 & 18,8 \\
\hline 2. glyphosate ${ }^{1}$ a $400 \mathrm{~mL} \mathrm{ha}^{-1}$ & 38,5 & 45,3 & 39,3 & 24,0 & 22,5 & 20,8 \\
\hline 3. glyphosate ${ }^{1}$ a $200 \mathrm{~mL} \mathrm{ha}^{-1}+$ sulfometuron-methyl ${ }^{2}$ a $10 \mathrm{~g} \mathrm{ha}^{-1}$ & 37,0 & 37,5 & 37,0 & 24,3 & 22,8 & 20,3 \\
\hline 4. glyphosate ${ }^{1}$ a $150 \mathrm{~mL} \mathrm{ha}^{-1}+$ sulfometuron-methyl $^{2}$ a $12 \mathrm{~g} \mathrm{ha}^{-1}$ & 34,3 & 39,3 & 36,8 & 23,8 & 22,0 & 19,3 \\
\hline 5. sulfometuron-methyl ${ }^{2}$ a $20 \mathrm{~g} \mathrm{ha}^{-1}$ & 34,3 & 40,3 & 35,8 & 23,5 & 21,0 & 19,8 \\
\hline 6. Testemunha & 33,5 & 37,3 & 32,3 & 24,5 & 22,0 & 20,3 \\
\hline $\mathrm{F}$ tratamento & $3,980^{*}$ & $2,65^{*}$ & $0,929 *$ & $0,929^{\mathrm{ns}}$ & $0,934^{\text {ns }}$ & $0,941^{\text {ns }}$ \\
\hline F bloco & 3,60 & 1,75 & 1,38 & 1,38 & 1,74 & 1,53 \\
\hline $\mathrm{CV}(\%)$ & 14,8 & 8,86 & 8,33 & 11,68 & 16,0 & 15,6 \\
\hline DMS & 5,69 & 5,2 & 3,31 & 4,12 & 5,17 & 4,58 \\
\hline
\end{tabular}

* Significativo a $5 \%$ de probabilidade pelo teste $\mathrm{T}$.
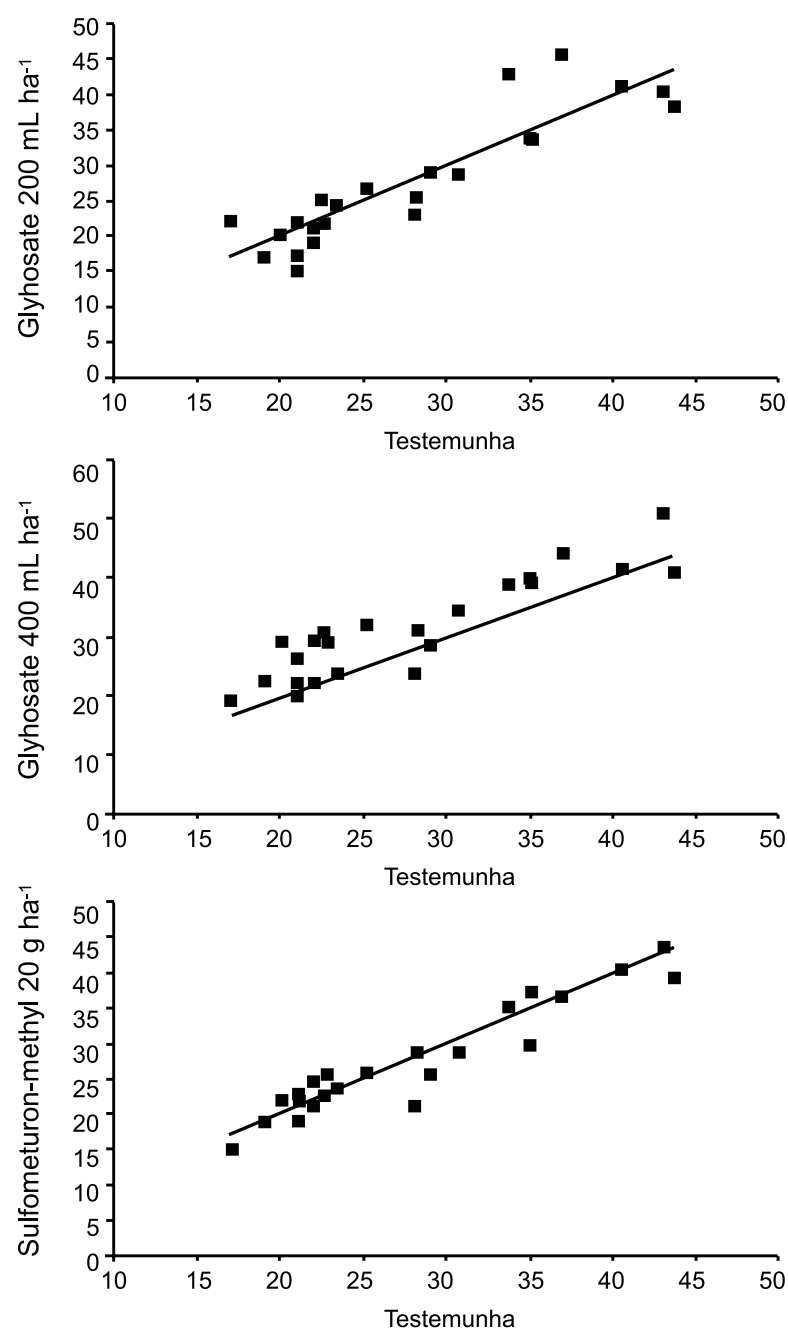

Figura 2 - Número de perfilhos da cana-de-açúcar, quando comparada com glyphosate $400 \mathrm{~mL} \mathrm{ha}^{-1}$ e $200 \mathrm{~mL} \mathrm{ha}^{-1}$ e sulfometuron-methyl $20 \mathrm{~g} \mathrm{ha}^{-1}$, em relação à testemunha. Igaraçu do Tiete-SP, 2007. tecnológica da cultura, embora não tenham alterado significativamente a produtividade.

O crescimento da cana-de-açúcar sofreu interferência no início do seu desenvolvimento inicial (90 DAC), quando submetido a doses mais elevadas dos produtos estudados. Doses entre 200 e $150 \mathrm{~mL} \mathrm{ha}{ }^{-1}$ de glyphosate e entre 12 e $10 \mathrm{~g}$ de sulfometuron-methyl não interferem nas características de crescimento da cultura e podem melhorar a qualidade tecnológica.

Pelos dados observados, é possivel inferir que tanto o glyphosate como o sulfometuronmethyl alteram a qualidade e a produção da cana-de-açúcar, com reflexos positivos na margem de produção agrícola. Os efeitos metabólicos desses produtos na planta se estendem após a colheita, promovendo alterações no desenvolvimento e crescimento da cultura.

\section{LITERATURA CITADA}

ALMEIDA, J. C. V. et al. Eficiência agronômica de sulfometuronmethil como maturador na cultura da cana-deaçúcar. STAB, v. 21, n. 3, p. 36-37, 2003.

BARBIERI, J. 0s anos do Proálcool no centro do debate. Jornal da Unicamp, novembro de 2005. Disponível em: http://www.unicamp.br/unicamp/unicamp_hoje/ju/ novembro2005/ju309pag11.html

BARBOSA, M. H. P. et al. Variedades melhoradas de cana-deaçúcar para Minas Gerais. Inf. Agropec., v. 28, p. 20-24, 2007.

CASAGRANDE, A. A. Tópicos de morfologia e fisiologia da cana-de-açúcar. Jaboticabal: Funep, 1991. 157 p. 
CAPUTO, M. M. et al. Resposta de genótipos de cana-deaçúcar a aplicação de indutores da maturação. Bragantia, v. 67, n. 12, p. $15-23,2008$.

CASTRO, P. R. C. et al. Ação comparada de ethrel, fuzilade e roundup, em duas épocas de aplicação, na maturação e produtividade da cana-de-açúcar 'SP 70-1143'. R. Agric., v. 77, n. 1, p. 23-38, 2002.

CASTRO, P. R. C.; CHRISTOFFOLETI, P. J. Fisiologia da cana-de-açúcar. In: MENDONÇA, A. C. Cigarrinhas da cana-de-açúcar: controle biológico. Maceió: Insecta, 2005. p. 3-48.

CONSECANA. Manual de instrução. 4.ed. Piracicaba: Conselho dos Produtores de Cana-de-Açúcar/Açúcar e álcool do estado de São Paulo, 2003. 115 p.

FERNANDES, A. C. Cálculos na agroindústria da canade-açúcar. Piracicaba: STAB, 2003. 240 p.

LEITE, G. H. P. et al. Qualidade tecnológica, produtividade e margem de contribuição agrícola da cana-de-açúcar em função da aplicação de reguladores vegetais no início da safra.

Ci. Rural, v. 39, n. 3, p. 726-732, 2009.

LIANG, X. et al. The 1-aminociclopropane-1-carboxilate synthase genmne family of Arabidopsis thaliana. Proc. Nalt Acad. Sci., v. 89, p. 11046-11050, 1992.
MILHOLLON, R. W.; LEGENDRE, B. L. Influence of ethephon on plant population and yield of sugarcane (Saccharum spp. hybrids). Plant Growth Reg. Soc. Am., v. 23, n. 1, p. 17-30, 1995.

OLIVEIRA, M. W. et al. Lixiviação de nitrogênio, potássio, cálcio e magnésio em solo arenoso cultivado com cana-deaçúcar. Pesq. Agropec. Bras., v. 37, n. 6, p. 861-868, 2002.

ROMERO, E. R. et al. Actualizacion de las recomendación de manejo de glifosato como madurador de la caña de azucar. Avance Agroind., v. 21, n. 22, p. 22-27, 2000

SHETIYA, H. L.; DENDSAY, J. P. S. Morpho-physiological and quality traits in sugarcane in response to post emergence treatment with 2-chloroethyl-phosphonic acid. Indian Sugar, v. 41, n. 1, p. 37-40, 1991

SILVA, M. M. et al. Uso de reguladores de crescimento como potencializadores do perfilhamento e da produtividade em cana-soca. Bragantia, v. 66, n. 4, p. 545-552, 2007.

VIANA, R. S. et al. Efeito da aplicação de maturadores químicos na cultura da cana de açúcar. Acta Sci. Agron., v. 30, n. 1, p. 65-71, 2008.

WIEDENFELD, B. Enhanced sugarcane establishment using plant growth regulators. J. Am. Soc. Sugar Cane Technol., v. 23, n. 2 , p. $48-61,2003$. 